\title{
Evaluation of the efficacy of Hylotelephium purpureum gel in the treatment of experimental periodontitis
}

\author{
XIAOFENG WANG ${ }^{1}$, WEI WANG ${ }^{1}$, WEI LI $^{2}$, ZHIDUO NIU $^{2}$, QING WANG $^{2}$, \\ DIANWEN ZHANG ${ }^{2}$, HUI LI ${ }^{1}$ and JINCHENG WANG ${ }^{3}$ \\ ${ }^{1}$ Department of Stomatology, China Japan Union Hospital, Jilin University, Changchun, Jilin 13033; \\ ${ }^{2}$ Academy of Traditional Chinese Medicine, Changchun, Jilin 130021; ${ }^{3}$ Orthopedic Department, \\ Second Hospital of Jilin University, Changchun, Jilin 130041, P.R. China
}

Received January 20, 2016; Accepted December 15, 2017

DOI: 10.3892/br.2018.1065

\begin{abstract}
Local drug delivery systems have been proposed for the treatment of periodontitis, and Hylotelephium purpureum may be a suitable agent for use in such a system. The present study aimed to formulate a Hylotelephium purpureum gel (HPG) and evaluate the anti-inflammatory activity and antinociceptive effects of Hylotelephium purpureum and to assess the duration of action and efficacy of HPG in the treatment of experimental periodontitis in a KM mouse model. First, an acute toxicity study was performed, and secondly, xylene-induced mouse-ear edema, acetic acid-induced mouse peritoneal capillary permeability and carrageenan-induced hind-paw edema tests were used to investigate the anti-inflammatory activity of the gel. The acetic acid-induced writhing response and hot-plate tests were used to evaluate the antinociceptive activity. The therapeutic effects of HPG in experimental periodontitis were evaluated and minimum inhibitory concentration of the gel was determined. The results showed that intragastrically administration of $80 \mathrm{ml} / \mathrm{kg}$ of HPG produced no toxic effects. There were statistically significant changes in gingival indexes and sulcus bleeding indexes in the high-dose HPG-treated group. The serum levels of superoxide dismutase and glutathione peroxidase were significantly heightened, while the level of malondialdehyde was decreased. HPG exhibited $32.7 \%$ inhibition of edema, and altered the peritoneal capillary permeability in mice. The gel had relatively good bacteriostatic and bactericidal effects. It also exhibited antinociceptive activity, as demonstrated by the acetic acid-induced writhing response
\end{abstract}

Correspondence to: Dr Wei Wang, Department of Stomatology, China Japan Union Hospital, Jilin University, 126 Xiantai Street, Changchun, Jilin 13033, P.R. China

E-mail: xiaofeng2238@sina.com

Dr Jincheng Wang, Orthopedic Department, Second Hospital of Jilin University, 218 Ziqiang Street, Changchun, Jilin 130041, P.R. China E-mail: jinchengwang@hotmail.com

Key words: anti-inflammatory activity, Hylotelephium purpureum gel, experimental periodontitis, antinociceptive activity test and hot-plate test. In summary, the present study demonstrates that HPG is effective in the treatment of experimental periodontitis.

\section{Introduction}

Periodontitis is a common disease, with 5-30\% prevalence in the adult population (1). Periodontitis is an inflammatory process of the periodontal tissues caused by bacterial infection, which results in the destruction of periodontal connective tissue and the reabsorption of the alveolar bone (2). Hylotelephium purpureum (also known as Sedum purpureum) grows in the Far East, Japan, Europe, North America and Northeast China (3). The grass is a herbal cure in traditional Chinese medicine due to its anti-inflammatory, analgesic, antispasmodic, antipyretic, antimicrobial, and antioxidant properties $(4,5)$. However, its efficacy in the treatment of periodontal diseases has not yet been elucidated.

Based on previously reported favorable aspects of Hylotelephium purpureum, we hypothesized that it would be a beneficial antioxidant agent for the suppression of periodontal inflammation and alveolar-bone destruction in periodontal disease. Therefore, the present study aims to investigate the anti-inflammatory activity and antinociceptive effects of a gel form of the plant, and to assess the duration of activity and efficacy of a Hylotelephium purpureum gel (HPG) in the treatment of experimental periodontitis in a Chinese Kun Ming (KM) mouse model.

\section{Materials and methods}

Preparation of HPG. A total of $10 \mathrm{~g}$ of Hylotelephium purpureum whole grass extract was soaked in $500 \mathrm{ml}$ of purified water, neutralized to a $\mathrm{pH}$ of $8-9$, and then dissolved. Following this, $160 \mathrm{~g}$ of poloxamer 407 was added to the filtrated drug solution. Distilled water was then added to bring the quantity of the solution to $1,000 \mathrm{ml}$. The gel was stored at ambient temperature. The HPG formulation was prepared by the Academy of Traditional Chinese Medicine (Changchun, China).

Animals. KM mice (nulliparous, 8-12 weeks) and Wistar rats (5-10 weeks) of both sexes were obtained from the Changchun 
Institute of Biological Products Co., Ltd. (Changchun, China). The experimental procedures of the present study were approved by the Animal Ethics Committee. The animals were housed in a well-ventilated animal house with a $12 \mathrm{~h}$ light and dark schedule and easy access to water and a standard pellet diet. Animals were randomly selected, marked to permit individual identification, and kept in their cages for at least 5-7 days prior to dosing to allow for acclimatization to the laboratory conditions.

Acute toxicity experiment. Forty KM mice were randomly divided into two groups of $20 \mathrm{mice} /$ group (10 males and 10 females) and were used for the acute oral toxicity study. Drinking water and food were provided throughout the experiment, except for a short fasting period wherein drinking water was still provided ad libitum, but no food was provided for $16 \mathrm{~h}$ prior to treatment. A single high dose of $40 \mathrm{ml} / \mathrm{kg}$ of $1.0 \%$ HPG was intragastrically administered to mice in the treatment group. Meanwhile, the second group of mice were allotted distilled water and were regarded as the control group. All of the animals were weighed and visually observed for mortality, behavioral patterns, changes in physical appearance, injury, pain, or other signs of illness daily for 14 days (6). At the end of the acute toxicity study, all mice were sacrificed. Vital organs such as the heart, kidneys, liver, lung and spleen were isolated and examined for any lesions. All of the individual organs were weighed, and their features were compared between both the treated and control groups.

Determination of maximum tolerance. Forty KM mice of both sexes were randomly divided into two groups: An HPG-treated group and a control group. Mice in the HPG-treated group were intragastrically administered HPG at a maximal dose of $40 \mathrm{ml} / \mathrm{kg}$, twice a day at a $4 \mathrm{~h}$ interval (total of $80 \mathrm{ml} / \mathrm{kg}$ per day), while the control group received an equal volume of deionized water for 14 days. Following administration of HPG or water, the responses of the mice, including toxic reactions and mortality, were observed and recorded each successive day for a 2-week period. At the end of the experiment, animals were sacrificed for gross-anatomy checks. Evaluations and recordings were conducted to determine whether there were any obvious changes in major organs under macroscopic observation.

Experimental periodontitis. A total of 50 Wistar albino rats (5-10 weeks old) weighing 150-250 g were used in the present study. The animals were anesthetized with ketamine. Preperiodontal examinations were conducted, and the upper second molars were ligated using a 4-0 sterile braided-silk suture (Hangzhou Westlake Biological Materials Co., Ltd), which was pretreated with Porphyromonas gingivalis. Soft tissue indicators were measured. Four weeks later, two rats were taken at random and sacrificed. Histological examinations of the maxillary molars and their periodontal tissues confirmed the model was established successfully (7).

Evaluation of the therapeutic effects of experimental periodontitis. The remaining rats were divided into 6 groups, each containing 8 animals: A non-ligated group; a ligature only group; a ligature plus treatment with standard group ( $\mathrm{Su} \mathrm{XiaO}$ Ya Tong Ning Ding, which is a proprietary Chinese medicine for the treatment of stomatal toothache, dental caries and chronic pulpitis); and three ligature plus treatment with HPG groups (1.0, 0.5 and $0.25 \%$, twice a day for 2 weeks). All rats were anesthetized following 2 weeks. We recorded the observed gingival index (GI) and gingival sulcus-bleeding index in each animal, as previously described $(8,9)$. Additionally, serum superoxide dismutase (SOD), glutathione peroxidase (GPx) and malondialdehyde (MDA) levels were measured $(10,11)$. Alveolar bone loss in the first molars was determined histologically. Periodontal tissues were histopathologically examined to assess any differences among the study groups.

The following classifications were used to score periodontal tissue inflammation: 0 , no inflammation; 1 , periodontal membrane vascular hyperemia, bleeding, mild inflammatory cell infiltration, osteoclasts occasionally visible or not present; 2, periodontal ligament vascular obviously dilated and congested, bleeding, inflammatory cells with moderate infiltration, membrane of increased width or partial disappearance, visible as a few osteoclasts, and mild alveolar bone absorption; and 3, weak tooth film, inflammatory cells and severe infiltration, abscess formation, broken bone cell infiltration, cementum and an alveolar bone with obvious absorption.

\section{Anti-inflammatory activity}

Xylene-induced mouse-ear edema test. A total of $50 \mathrm{KM}$ mice were randomly divided into five groups of 10 each, as follows: Blank control group (equal volume of saline); a positive control (treatment with standard Su Xiao Ya Tong Ning Ding); and three groups receiving treatment with HPG at various concentrations $(1.0,0.5$ and $0.25 \%)$. Mice in each group were intragastrically administered at the design dose (capacity of $0.2 \mathrm{ml} / 10 \mathrm{~g}$ ) for 7 consecutive days. After the last administration, $0.05 \mathrm{ml}$ of xylene was evenly applied on the right ear of each mouse, and the left ear served as the control. After $45 \mathrm{~min}$, the mice were euthanized and the left and right ears were removed, round ear samples in the corresponding parts were removed with a $4 \mathrm{~mm}$ radius punch, and the ears were weighed on an electronic balance. The degree of edema was recorded as the weight of the right ear sample subtracted from the weight of left earpiece. The degree of edema among the various groups was compared, and the edema inhibition rate was calculated: Edema inhibition rate $=($ degree of edema of blank control group - degree of edema of treatment group)/degree of edema of blank control group x $100 \%$.

Effects on acetic acid-induced mouse peritoneal capillary permeability. A total of $50 \mathrm{KM}$ mice were randomly divided into five groups of 10 each: A blank control group (equal volume of saline); a positive control group (treatment with standard Su Xiao Ya Tong Ning Ding); and three treatment with HPG groups (1.0, 0.5 and $0.25 \%)$. Treatment groups were intragastrically administered $(0.2 \mathrm{ml} / 10 \mathrm{~g})$ for 7 consecutive days. The blank control group was given an equivalent volume of distilled water. After the last administration, the mice were given a tail-vein injection of $0.1 \mathrm{ml} / 10 \mathrm{~g}$ of $0.5 \%$ Evans Blue solution in saline, as well as a $0.2 \mathrm{ml}$ intraperitoneal injection of $0.6 \%$ acetic acid per mouse. Thirty minutes later, the animals were sacrificed, and the abdominal skin and muscle were removed. The abdominal cavity was washed with $6 \mathrm{ml}$ of a $0.9 \% \mathrm{NaCl}$ solution; the washing liquid was pipetted out and combined. Then, a $0.9 \% \mathrm{NaCl}$ solution was added to bring the 
Table I. Comparisons of the gingival and sulcus bleeding indexes in response to treatment.

\begin{tabular}{|c|c|c|c|c|}
\hline \multirow[b]{2}{*}{ Group } & \multicolumn{2}{|c|}{ GI } & \multicolumn{2}{|c|}{ SBI } \\
\hline & Before & After & Before & After \\
\hline Non-ligated & 0 & 0 & 0 & 0 \\
\hline Ligature alone & $2.75 \pm 0.46$ & $3.00 \pm 0.00$ & $3.25 \pm 1.04$ & $3.38 \pm 1.19$ \\
\hline Ligature + Su Xiao Ya Tong Ning Ding & $2.63 \pm 0.52$ & $1.13 \pm 0.64^{\mathrm{c}}$ & $3.50 \pm 1.41$ & $2.00 \pm 1.07^{\mathrm{a}}$ \\
\hline Ligature $+1.0 \% \mathrm{HPG}$ & $2.50 \pm 0.76$ & $0.75 \pm 0.71^{\mathrm{c}}$ & $3.50 \pm 1.20$ & $1.50 \pm 1.20^{\mathrm{b}}$ \\
\hline Ligature $+0.5 \%$ HPG & $2.50 \pm 0.53$ & $1.13 \pm 0.64^{\mathrm{c}}$ & $3.38 \pm 1.19$ & $2.13 \pm 1.13^{\mathrm{a}}$ \\
\hline Ligature $+0.25 \%$ HPG & $2.50 \pm 0.53$ & $1.50 \pm 0.53^{\mathrm{b}}$ & $3.13 \pm 1.13$ & $2.13 \pm 0.83$ \\
\hline
\end{tabular}

P-values were calculated by comparing values with the pre-treatment group. ${ }^{a} \mathrm{P}<0.05 ;{ }^{b} \mathrm{P}<0.01 ;{ }^{\mathrm{c}} \mathrm{P}<0.001$. GI, gingival index; SBI, sulcus bleeding index; HPG, Hylotelephium purpureum gel.

volume to $10 \mathrm{ml}$, followed by centrifugation at $500 \mathrm{x}$. The supernatant was collected, and the absorbance was measured at $590 \mathrm{~nm}$. Differences among groups were compared.

Effect on carrageenan-induced hind-paw edema. The KM mice were randomly divided into five groups, as described previously. Local treatment was conducted with saline, standard drug (Su Xiao Ya Tong Ning Ding ${ }^{\circledR}$ ) and HPG (1.0, 0.5 and $0.25 \%$ ) twice a day for 5 days. At $1 \mathrm{~h}$ following the last administration, the mice were administered a subcutaneous injection of $0.1 \mathrm{ml}$ of a $1 \%$ solution of carrageenan into the plantar side of the left-hind paw (12). Local treatment was conducted again on the injection site. The thickness of the dorsoventral diameter in each animal was measured using a pair of dial-thickness gauge calipers at 1,2 and $4 \mathrm{~h}$ following the induction of inflammation.

\section{Antinociceptive analysis}

Acetic-acid induced writhing response. KM mice were locally treated with their respective treatments as previously described in material and method twice a day for 5 days. After $1 \mathrm{~h}$ treatment, $0.7 \%$ acetic acid $(0.1 \mathrm{ml} / 10 \mathrm{~g}$ body weight $)$ was administered intraperitoneally to each mouse. The mice were observed, and the number of abdominal constrictions and stretching over a period of 5-15 min were counted.

Hot-plate test. HPG (1.0, 0.5 and $0.25 \%)$ was administered dermally for 5 days. Following the last administration, mice were individually placed on a heated plate at $55 \pm 1^{\circ} \mathrm{C}$. The latency time of forepaw licking or jumping was determined at 60,120 and $240 \mathrm{~min}$ following treatment.

Determination of minimum inhibitory concentration (MIC). The MICs of HPG, tinidazole and Su Xiao Ya Tong Ning Ding against five bacterial strains were determined by the test-tube continuous dilution method. HPG was serially diluted at concentrations of 5.0, 2.5, 1.25, 0.62, $0.31,0.15$ and $0.08 \mathrm{mg} / \mathrm{ml}$. The concentrations of tinidazole in the medium was 128,64 , $32,16,8,4,2$ and $1 \mu \mathrm{g} / \mathrm{ml}$. The concentrations of Su Xiao Ya Tong Ning Ding were 30, 15, 7.5, 3.7, 1.85 and $0.92 \%$. A total of $0.05 \mathrm{ml}$ of a bacterial solution was added to each group and cultured at $37^{\circ} \mathrm{C}$ for $18 \mathrm{~h}$, and a blank control was used. The concentration of drug in the last clear test tube was taken as the minimum inhibitory concentration.

Statistical analysis. The SPSS 17.0 software (SPSS, Inc., Chicago, IL, USA) was used to analyse and process the data. All data are represented as the means \pm SD of three independent experiments. Statistical significance was tested by Student's t-test and one-way analysis of variance. $\mathrm{P}<0.05$ was considered to represent a statistically significant difference.

\section{Results}

HPG analysis. HPG containing a flavonoid extract was formulated as a local delivery drug. The $\mathrm{pH}$ was within the acceptable range of 7.0-8.0, even at the end of 30 days. The product contained a total amount of quercetin $\left(\mathrm{C}_{15} \mathrm{H}_{10} \mathrm{O}_{7}\right)$ and rhizoma kaempferiae $\left(\mathrm{C}_{15} \mathrm{H}_{10} \mathrm{O}_{6}\right)$ was $>5.0 \mathrm{mg} / \mathrm{ml}$, as evaluated by chromatograph (data not shown).

Acute toxicity and maximum tolerance. In the acute toxicity test, administration of HPG $(40 \mathrm{ml} / \mathrm{kg})$ to mice did not cause death or acute behavioral changes during the observation periods, and we did not notice any pathological changes in the mice. The $\mathrm{LD}_{50}$ was estimated to be $>40 \mathrm{ml} / \mathrm{kg}$. For maximum tolerance, a further test of HPG did not demonstrate any behavioral changes or mortality in mice at doses of $80 \mathrm{ml} / \mathrm{kg}$ during the 14 days of the experiment. HPG was safe at the given dose in mice.

The therapeutic effects of HPG in experimental periodontitis GI and sulcus bleeding index (SBI). The mean GI prior to treatment in the control, Su Xiao Ya Tong Ning Ding, and 1.0, 0.5 and $0.25 \% \mathrm{HPG}$ groups were $2.75 \pm 0.46,2.63 \pm 0.52,2.50 \pm 0.76$, $2.50 \pm 0.53$ and $2.50 \pm 0.53$, respectively. The mean GIs after 14 days in each group were $3.00 \pm 0.00,1.13 \pm 0.64,0.75 \pm 0.71$, $1.13 \pm 0.64$ and $1.50 \pm 0.53$, respectively. The mean percentage changes were $-9.09,57.03,70.00,54.80$ and $40.00 \%$, respectively. There was a statistically significant change in the GI at the end of 14 days $(\mathrm{P}=0.001$; Table $\mathrm{I})$. As shown in Table I, the mean sulcus bleeding index prior to treatment in the control, Su Xiao Ya Tong Ning Ding, and 1.0, 0.5 and 0.25\% HPG groups were $3.25 \pm 1.04,3.50 \pm 1.41,3.50 \pm 1.20,3.38 \pm 1.19$ and $3.13 \pm 1.13$, respectively. The mean SBIs at the end of 14 days 
Table II. Comparisons of the serum levels of SOD, GPx and MDA in different treatment groups.

\begin{tabular}{lcrr}
\hline Treatment group & SOD $(\mathrm{U} / \mathrm{ml})$ & GPx $(\mathrm{U} / \mathrm{ml})$ & $\mathrm{MDA}(\mathrm{nmol} / \mathrm{l})$ \\
\hline Non-ligated & $103.9 \pm 3.948$ & $196.2 \pm 6.735$ & $3.741 \pm 0.691$ \\
Ligature alone & $91.10 \pm 4.102$ & $157.27 \pm 31.48$ & $8.928 \pm 1.003$ \\
Ligature + Su Xiao Ya Tong Ning Ding & $99.84 \pm 6.377^{\mathrm{b}}$ & $190.34 \pm 5.101^{\mathrm{a}}$ & $6.231 \pm 1.099^{\mathrm{c}}$ \\
Ligature + 1.0\% HPG & $99.11 \pm 5.112^{\mathrm{b}}$ & $188.48 \pm 5.834^{\mathrm{a}}$ & $6.570 \pm 1.015^{\mathrm{b}}$ \\
Ligature + 0.5\% HPG & $93.77 \pm 5.626$ & $187.55 \pm 15.81^{\mathrm{a}}$ & $7.133 \pm 0.778^{\mathrm{b}}$ \\
Ligature + 0.25\% HPG & $92.174 \pm 5.2005$ & $182.70 \pm 19.69$ & $7.949 \pm 1.495$
\end{tabular}

$\mathrm{P}$-values were calculated by comparing values with the pre-treatment group. ${ }^{\mathrm{a}} \mathrm{P}<0.05$; ${ }^{\mathrm{b}} \mathrm{P}<0.01$; ${ }^{\mathrm{c}} \mathrm{P}<0.001$. HPG, Hylotelephium purpureum gel; SOD, superoxide dismutase; GPx, glutathione peroxidase; MDA, malondialdehyde.

Table III. Inhibitory effect of HPG on xylene-induced ear edema in mice.

\begin{tabular}{lccc}
\hline Treatment group & Dose $(\mathrm{ml})$ & Degree of ear edema $(\mathrm{mg})$ & Edema inhibition rate $(\%)$ \\
\hline Ligature alone & N/A & $18.7 \pm 2.79$ & 85.3 \\
Su Xiao Ya Tong Ning Ding & 0.3 & $11.2 \pm 4.42^{\mathrm{b}}$ & $42.6^{\mathrm{c}}$ \\
$1 \%$ HPG & 0.3 & $7.60 \pm 4.72^{\mathrm{c}}$ & $32.7^{\mathrm{c}}$ \\
$0.5 \%$ HPG & 0.3 & $10.3 \pm 3.92^{\mathrm{c}}$ & $46.8^{\mathrm{c}}$ \\
$0.25 \%$ HPG & 0.3 & $14.5 \pm 4.95^{\mathrm{a}}$ & $63.5^{\mathrm{a}}$ \\
\hline
\end{tabular}

P-values were calculated by comparing values with the pre-treatment group. ${ }^{a} \mathrm{P}<0.05$; ${ }^{b} \mathrm{P}<0.01 ;{ }^{\mathrm{c}} \mathrm{P}<0.001$. HPG, Hylotelephium purpureum gel.

in each group were $3.38 \pm 1.19,2.00 \pm 1.07,1.50 \pm 1.20,2.13 \pm 1.13$ and $2.13 \pm 0.83$, and the mean percentage changes were -4.00 , $42.86,57.14,36.98$ and $31.95 \%$, respectively. There was a statistically significant difference in the SBI at the end of 14 days $(\mathrm{P}=0.01)$.

Serum levels of SOD, GPX and MAD. The serum levels of SOD in the non-ligated, ligature alone, Su Xiao Ya Tong Ning

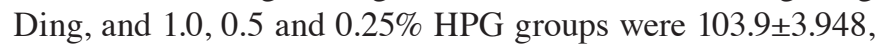
$91.10 \pm 4.102,99.84 \pm 6.377,99.11 \pm 5.112,93.77 \pm 5.626$ and $92.174 \pm 5.2005$, respectively. The GPx levels in the different groups were $196.2 \pm 6.735,157.27 \pm 31.48,190.34 \pm 5.101$, $188.48 \pm 5.834,187.55 \pm 15.81$ and $182.70 \pm 19.69$. The MDA levels were $3.741 \pm 0.691,8.928 \pm 1.003,6.231 \pm 1.099,6.570 \pm 1.015$, $7.133 \pm 0.778$ and $7.949 \pm 1.495$. As shown in Table II, the levels of SOD and GPx in the Su Xiao Ya Tong Ning Ding group and $1 \%$ HPG group were significantly heightened $(\mathrm{P}<0.01, \mathrm{P}<0.05)$. However, the levels of MDA in these groups were decreased $(\mathrm{P}<0.001, \mathrm{P}<0.01)$. There were no statistically significant differences in the serum levels of SOD, GPx and MDA in the $0.25 \%$ HPG group compared with the ligature alone group.

Exterior behavioral observations and histopathological examinations. The animals in the non-ligated group increased gradually in weight, with normal diet and activity. Rats in the ligature-only group gradually showed loss of appetite and weight. While after a 14-day administration, the rats were gradually restored to activity and appetite in the administration groups, and the weight of these rats also increased. In the Su Xiao Ya Tong Ning Ding group, periodontal tissue was found to have inflammatory-cell infiltration, but the rate was significantly alleviated compared with the ligature-only
Table IV. Inhibitory effects of HPG on acetic acid-induced peritoneal capillary permeability.

\begin{tabular}{lcc}
\hline Treatment group & Dose $(\mathrm{ml})$ & Absorbance \\
\hline Non-ligated & N/A & $0.048 \pm 0.020^{\mathrm{c}}$ \\
Ligature alone & N/A & $0.421 \pm 0.093$ \\
Su Xiao Ya Tong Ning Ding & 0.3 & $0.276 \pm 0.113^{\mathrm{b}}$ \\
$1.0 \%$ HPG & 0.3 & $0.226 \pm 0.103^{\mathrm{c}}$ \\
$0.5 \%$ HPG & 0.3 & $0.262 \pm 0.101^{\mathrm{b}}$ \\
$0.25 \%$ HPG & 0.3 & $0.301 \pm 0.119^{\mathrm{a}}$
\end{tabular}

P-values were calculated by comparing values with the pre-treatment group. ${ }^{\text {a }} \mathrm{P}<0.05$; ${ }^{\text {b }}<0.01$; ${ }^{c} \mathrm{P}<0.001$. HPG, Hylotelephium purpureum gel.

group (Fig. 1). The rats in the high-dose HPG group showed significant reductions in gingival inflammation and pocket depth (Fig. 1D-F). The periodontal bone loss difference was not statistically significant. These results indicate that HPG of 1.0 and $0.5 \%$ and Su Xiao Ya Tong Ning Ding can significantly reduce the degree of injury to periodontal tissue in an experimental mouse model of periodontitis.

Inhibitory effects of HPG on xylene-induced ear edema in mice. The HPG high- and medium-dosage groups and the $\mathrm{Su}$ Xiao Ya Tong Ning Ding group all antagonized xylene-induced mouse-ear edema, when compared to the blank control group. Following treatment with HPG at high- or medium-doses, or Su Xiao Ya Tong Ning Ding the degree of ear edema was 
A

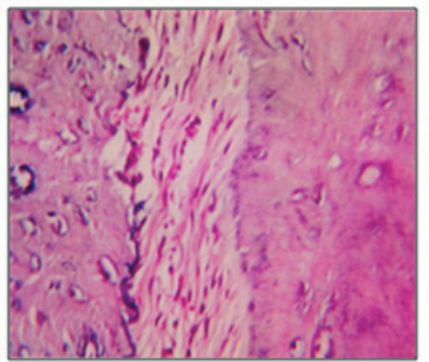

D

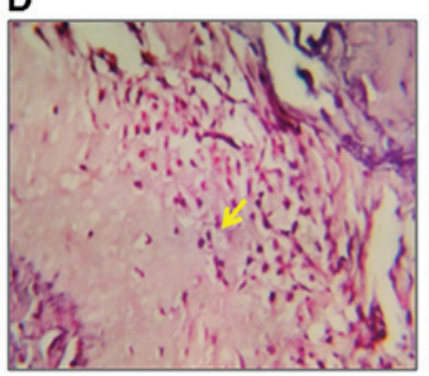

B

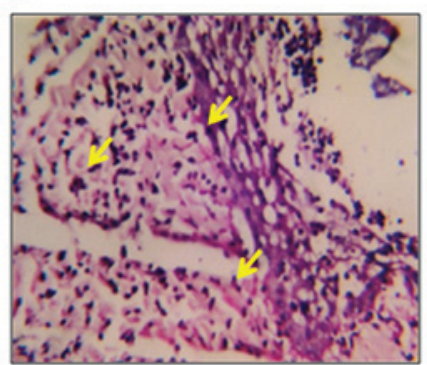

$\mathrm{E}$

C

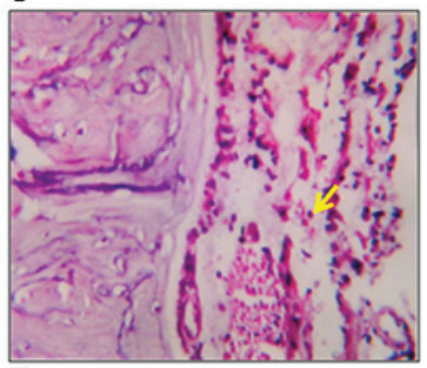

$F$

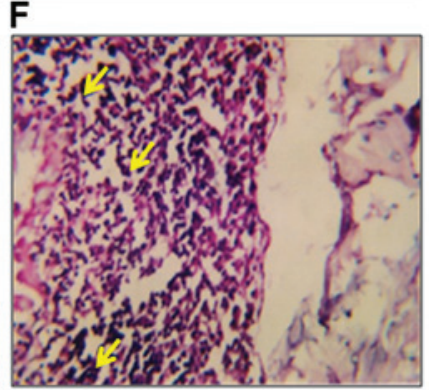

G

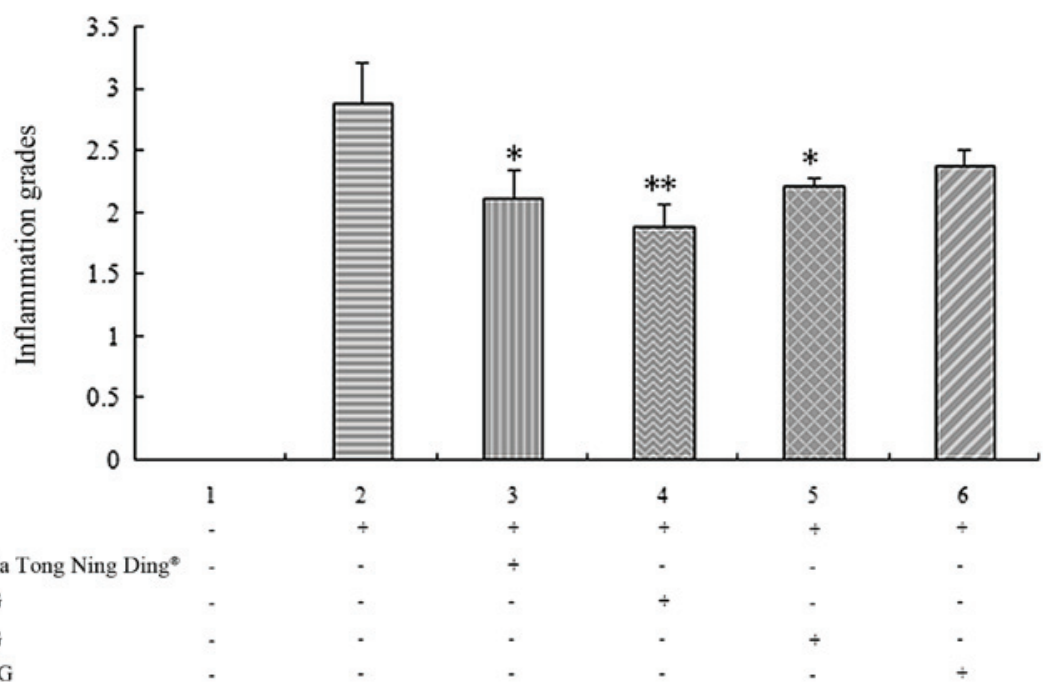

Figure 1. Periodontal inflammation grade in different treatment groups. (A) The histological images of the non-ligated group showing normal periodontium (magnification, x100). (B) The histological images of the ligature alone group, with intense inflammatory cell infiltrate, dilated blood vessels, and osteoclasts in their Howship's lacunae with multiple reabsorption foci (magnification, x100). (C) The histological images of Su Xiao Ya Tong Ning Ding with moderate inflammatory cell infiltrate in periodontal ligament and osteoclasts in their Howship's lacunae with multiple reabsorption foci. (D-F) Histological images of different doses of HPG (D, 1.0\%; E, 0.5\%; F, 0.25\%). Yellow arrows indicate inflammatory cells (magnification, x100). (G) Comparison of periodontal inflammation grade in different groups. HPG, Hylotelephium purpureum gel. ${ }^{*} \mathrm{P}<0.05 ;{ }^{* *} \mathrm{P}<0.01$.

markedly reduced, and the differences were statistically significant $(\mathrm{P}<0.01$ or $\mathrm{P}<0.05$; Table III).

Inhibitory effects of HPG on acetic-acid induced peritoneal capillary permeability in mice. Compared with the blank control group, the HPG high-, medium- and low-dosage groups and the Su Xiao Ya Tong Ning Ding group all had significantly inhibited $0.6 \%$-acetic-acid induced peritoneal capillary permeability in mice $(\mathrm{P}<0.05$ or $\mathrm{P}<0.01$; Table IV).

Evaluation of antinociceptive activity of $H P G$. As shown in Fig. 2, HPG exhibited antinociceptive activity in acetic acid-induced writhing response and hot-plate test. Local administration of the HPG (1.0, 0.5 and $0.25 \%)$ significantly decreased the number of writhes in mice induced by acetic acid, with inhibition rates of $57.25,34.35$ and $20.61 \%$, respectively, $(\mathrm{P}<0.01)$ in a dose-dependent manner. As the positive drug, Su Xiao Ya Tong Ning Ding produced a $67.18 \%$ reduction compared to the control. Meanwhile, compared to the control group at 60 and $120 \mathrm{~min}$, high-dose HPG could prolong the latency times of mice $(P<0.05$, $\mathrm{P}<0.01$, respectively). Su Xiao Ya Tong Ning Ding markedly increased the pain threshold of mice in the first $60 \mathrm{~min}$ $(\mathrm{P}<0.01)$, but it decreased it thereafter.

MIC determination results of HPG. The determination of the MIC of the different treatments is shown in Table V. The results demonstrate that HPG had relatively good bacteriostatic and bactericidal effects on Bacteroides melaninogenicus, Porphyromonas gingivalis, Fusobacterium nucleatum, Streptococcus mutans, Aggregatibacter actinomycetemcomitans and Bacteroides melaninogenicus. 
Table V. Minimum inhibitory concentrations of HPG, tinidazole and Su Xiao Ya Tong Ning Ding among bacterial species.

Minimum inhibitory concentration

Bacterial species

$1 \% \mathrm{HPG}(\mathrm{mg} / \mathrm{ml})$

Tinidazole $(\mu \mathrm{g} / \mathrm{ml})$

Su Xiao Ya Tong Ning Ding (\%)

Aggregatibacter actinomycetemcomitans 1

\begin{tabular}{|c|c|c|}
\hline 5.00 & 64 & 15.0 \\
\hline 2.50 & 32 & 15.0 \\
\hline 2.50 & 32 & 7.5 \\
\hline 2.50 & 16 & 7.5 \\
\hline 5.00 & 32 & 15.0 \\
\hline 2.50 & 16 & 7.5 \\
\hline 2.50 & 16 & 15.0 \\
\hline 1.25 & 8 & 7.5 \\
\hline 2.50 & 16 & 7.5 \\
\hline 5.00 & 64 & 15.0 \\
\hline 2.50 & 32 & 7.5 \\
\hline 2.50 & 32 & 7.5 \\
\hline 2.50 & 32 & 7.5 \\
\hline 5.00 & 64 & 15.0 \\
\hline 5.00 & 64 & 15.0 \\
\hline 5.00 & 64 & 15.0 \\
\hline 2.50 & 32 & 7.5 \\
\hline 1.25 & 16 & 3.7 \\
\hline 5.00 & 32 & 15.0 \\
\hline 5.00 & 32 & 15.0 \\
\hline 5.00 & 64 & 15.0 \\
\hline 2.50 & 32 & 15.0 \\
\hline 2.50 & 32 & 15.0 \\
\hline 2.50 & 32 & 15.0 \\
\hline 1.25 & 16 & 3.7 \\
\hline 2.50 & 16 & 3.7 \\
\hline 2.50 & 16 & 3.7 \\
\hline
\end{tabular}

Aggregatibacter actinomycetemcomitans 2

Aggregatibacter actinomycetemcomitans 3

Aggregatibacter actinomycetemcomitans 4

Aggregatibacter actinomycetemcomitans 5

Bacteroides melaninogenicus 1

Bacteroides melaninogenicus 2

Bacteroides melaninogenicus 3

Bacteroides melaninogenicus 4

Streptococcus mutans A

Streptococcus mutans $B$

Streptococcus mutans $C$

Streptococcus mutans $D$

Streptococcus mutans $E$

Streptococcus mutans $F$

Streptococcus mutans $G$

Porphyromonas gingivalis 1

Porphyromonas gingivalis 2

Porphyromonas gingivalis 3

Porphyromonas gingivalis 4

Porphyromonas gingivalis 5

Porphyromonas gingivalis 6

Porphyromonas gingivalis 7

Porphyromonas gingivalis 8

Fusobacterium nucleatum 1

Fusobacterium nucleatum 2

Fusobacterium nucleatum 3

HPG, Hylotelephium purpureum gel.
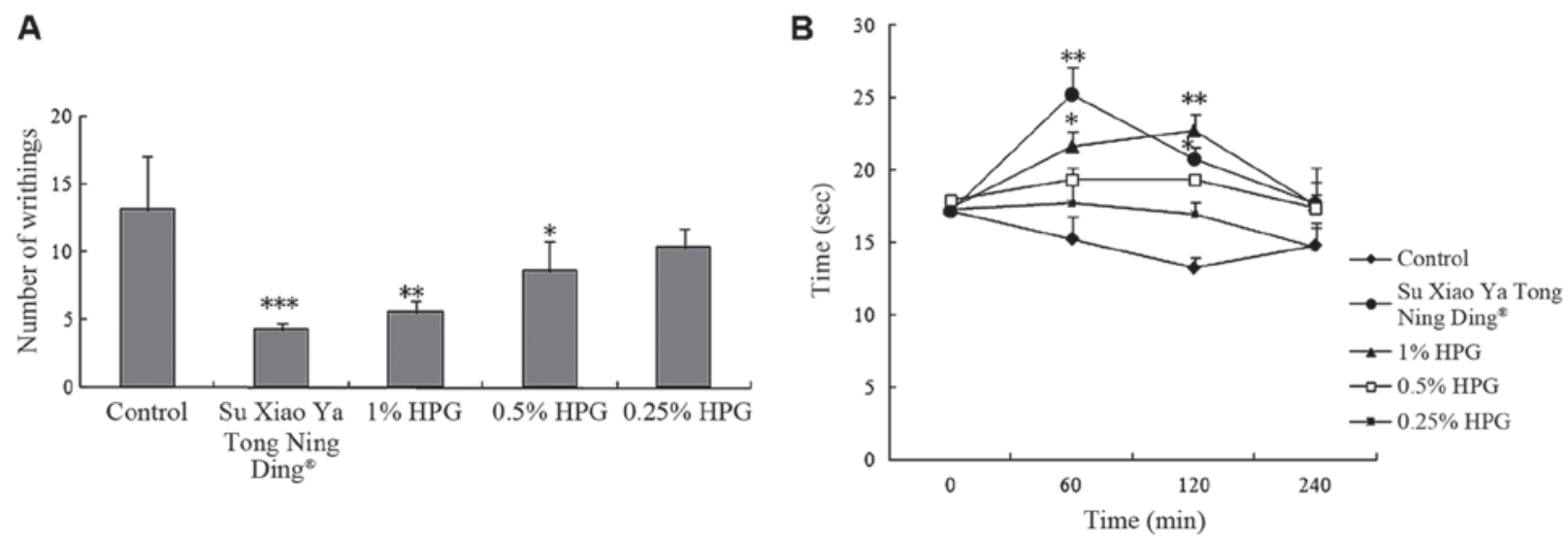

Figure. 2 Antinociceptive analysis of different treatment groups. (A) Acetic acid-induced writhing response. " $\mathrm{P}<0.05$; ${ }^{* *} \mathrm{P}<0.01$. (B) Hot-plate test. HPG, Hylotelephium purpureum gel. ${ }^{*} \mathrm{P}<0.05 ;{ }^{* *} \mathrm{P}<0.01 ;{ }^{* * *} \mathrm{P}<0.001$.

\section{Discussion}

Periodontitis is a chronic inflammatory disease caused by bacterial infection of the supporting tissues surrounding the teeth. The concept of local delivery of chemotherapeutic agents to the periodontal pocket as a method to treat 
periodontal disease has been studied for over the past few decades. Although various locally delivered antimicrobial agents are commercially available, the need for safe, effective, and economical agents has motivated the use of various natural extracts. Various herbal products and their extracts such as guava, pomegranate, neem, propolis, tulsi, green tea, cranberry, grapefruit, etc., in the form of mouthwashes and gels have shown significant advantages over the chemical ones in the treatment of periodontal diseases (13-15).

Periodontal disease can be induced in rodents by tying a ligature of 2-0-5-0 braided silk around the cervix of the maxillary or mandibular molars, or by injecting lipopolysaccharides into the papilla, or a combination of both (16). Souza et al (17) used a period of 4 weeks for periodontitis induction in the maxilla. This timeframe was similar to the study period used in our study.

Hylotelephium purpureum is an herbal cure in traditional medicine because of its anti-inflammatory, analgesic, antispasmodic, antipyretic, antimicrobial, and antioxidant properties $(4,5)$. However, its efficacy in the treatment of periodontal diseases has not yet been elucidated. In the present study, we squeezed the juice of the Hylotelephium purpureum from the whole grass, and then extracted and separated its effective ingredients, filtering the effective parts of the plant. We found that the extract contained $76 \%$ quercetin and kaempferide. Quercetin is a flavonol found in many fruits, vegetables, leaves and grains. Kaempferide is an O-methylated flavonol, a type of chemical compound. HPG was produced from the extract and used to investigate the anti-inflammatory activity and antinociceptive effects, as well as assessed the durations of the action and the efficacy of iHPG, in the treatment of experimental periodontitis in a KM mouse model.

In the present study, we formulated and evaluated the anti-inflammatory activity and antinociceptive effects of Hylotelephium purpureum and assessed the duration of action and efficacy of Hylotelephium purpureum in the treatment of experimental periodontitis. The results demonstrated that $H P G$ obviously changed the GI and SBI in our model of experimental periodontitis. The serum levels of SOD and GPx were significantly heightened, while the level of MAD was decreased. The gel showed $32.7 \%$ inhibition of edema, and it changed the peritoneal capillary permeability in mice. Meanwhile, it had relatively good bacteriostatic and bactericidal effects, as well as antinociceptive activity. Hence, HPG can be a useful adjunct to enhance the results of standard periodontal therapy.

In conclusion, within the limitations of the present study, HPG appears to be an attractive alternating agent that can be used for effective and safe local drug delivery as an adjunct to mechanical nonsurgical periodontal therapy.

\section{Acknowledgements}

The present study was supported by the grants from science and technology development projects of Jilin Province Department of Traditional Chinese Medicine (grant no. 20100919).

\section{References}

1. Miyazaki H, Pilot T, Leclercq MH and Barmes DE: Profiles of periodontal conditions in adults measured by CPITN. Int Dent J 41: 74-80, 1991.

2. Ryan ME: Nonsurgical approaches for the treatment of periodontal diseases. Dent Clin North Am 49: 611-636, vii, 2005.

3. Xinqi Chen HC, Dai L and Xia Z: The flora of China. Science Press. China, 2004.

4. Winekenstädde D, Angelis A, Waltenberger B, Schwaiger S, Tchoumtchoua J, König S, Werz O, Aligiannis N, Skaltsounis AL and Stuppner H: Phytochemical profile of the aerial parts of Sedum sediforme and anti-inflammatory activity of myricitrin. Nat Prod Commun 10: 83-88, 2015.

5. Sendl A, Mulinacci N, Vincieri FF and Wagner $\mathrm{H}$ : Anti-inflammatory and immunologically active polysaccharides of Sedum telephium. Phytochemistry 34: 1357-1362, 1993.

6. Peichl P: Health, safety and environmental protection in a biological research laboratory. Int Arch Occup Environ Health 73: S8-S13, 2000.

7. Liu R, Li N, Liu N, Zhou X, Dong ZM, Wen XJ and Liu LC: Effects of systemic ornidazole, systemic and local compound ornidazole and pefloxacin mesylate on experimental periodontitis in rats. Med Sci Monit 18: BR95-BR102, 2012.

8. Loe H and Silness J: Periodontal disease in pregnancy. I. Prevalence and severity. Acta Odontol Scand 21: 533-551, 1963.

9. Ainamo $\mathrm{J}$ and Bay I: Problems and proposals for recording gingivitis and plaque. Int Dent J 25: 229-235, 1975.

10. Shapira L, Gordon B, Warbington M and Van Dyke TE: Priming effect of Porphyromonas gingivalis lipopolysaccharide on superoxide production by neutrophils from healthy and rapidly progressive periodontitis subjects. J Periodontol 65: 129-133, 1994.

11. Borges I Jr, Moreira EA, Filho DW, de Oliveira TB, da Silva MB and Fröde TS: Proinflammatory and oxidative stress markers in patients with periodontal disease. Mediators Inflamm 2007: 45794, 2007.

12. Pereira SL, de Oliveira JW, Angelo KK, da Costa AM and Costa F: Clinical effect of a mouth rinse containing Ocimum gratissimum on plaque and gingivitis control. J Contemp Dent Pract 12: 350-355, 2011.

13. Kukreja BJDV: Herbal mouthwashes - a gift of nature. Int $\mathrm{J}$ Pharma Bio Sci 3: 46-52, 2012.

14. Desai AAM and Debnath S: A clinical trial to evaluate the effects of triphala as a mouthwash in comparison with chlorhexidine in chronic generalized periodontitis patient. IJDA Arch 2: 243-247, 2010.

15. Reddy PDST, Swarna LD and Purushothaman M: Local drug delivery of herbs for treatment of periodontitis. J Innov Trends Pharma Sci 1: 245-251, 2010.

16. Struillou X, Boutigny H, Soueidan A and Layrolle P: Experimental animal models in periodontology: A review. Open Dent J 4: 37-47, 2010.

17. Souza DM, Prado FA, Prado MA, Rocha RF and Carvalho YR: Evaluation of two morphometric methods of bone loss percentages caused by periodontitis in rats in different locations. J Appl Oral Sci 18: 493-497, 2010. 